\title{
Chronic Pain is Associated with Increased TrkA Immunoreactivity in Spinoreticular Neurons
}

\author{
Sophie Pezet, ${ }^{1}$ Brigitte Onténiente, ${ }^{1}$ Gaël Grannec, ${ }^{1}$ and Bernard Calvino ${ }^{1,2}$ \\ 1/nstitut National de la Santé et de la Recherche Médicale U421, Institut Mondor de Médecine Moléculaire, Faculté de \\ Médecine, F-94010 Créteil Cedex, France, and 2Faculté des Sciences, Université de Paris XII, F-94010 Créteil Cedex, \\ France
}

Repetitive noxious stimulation leads to permanent adaptive changes of central pathways involved in the genesis and integration of nociception. Several classes of neurotrophic factors that affect brain plasticity are also involved in the regulation of sensory functions in adulthood. To investigate a putative role of nerve growth factor (NGF) in central plasticity linked to chronic pain, modifications in immunoreactivity (IR) for the high-affinity NGF receptor, TrkA, were studied at spinal levels in a rat model of inflammatory chronic pain, adjuvant-induced arthritis (AIA). We report a specific increase in the number of TrkA-IR profiles in laminae $\mathrm{V}-\mathrm{VI}$ at lumbar levels $\mathrm{L} 3$ and $\mathrm{L} 4$ in arthritic rats. Tract tracing using FluoroGold injections in the ventrobasal complex of the thalamus and in the brainstem showed that these in- creased TrkA-IR profiles are spinoreticular neurons. Dual labeling with calcitonin gene-related peptide or substance $P$ showed that TrkA-IR neurons were mainly located in projection fields of small- to medium-sized primary afferent fibers, which convey nociceptive inputs. These results suggest that TrkA-containing neurons of the spinal dorsal horn participate in the first central relay of transmission of nociceptive information to supraspinal centers. Enhanced numbers of TrkA-IR neurons during AIA strongly support the hypothesis of a participation of NGF in adaptive mechanisms of central nociceptive pathways observed in chronic pain states.

Key words: nerve growth factor; NGF; TrkA; adjuvant-induced arthritis; substance P; CGRP; spinal cord; rat
Central neuronal plasticity has been proposed as a structural and molecular correlate of chronic pain (Coderre et al., 1993). The spinal dorsal horn $(\mathrm{DH})$ is the first relay of central nociceptive pathways. Nociceptive primary afferent fibers project onto both specific and nonspecific nociceptive neurons that convey information to supraspinal centers mainly located in the thalamus and brainstem (Jessel and Kelly, 1991). In several models of chronic pain, including arthritis, deep layers of the dorsal horn have been shown to be the site of a number of long-lasting molecular and electrophysiological changes, which affect both presynaptic and postsynaptic components of pain pathways (Coderre et al., 1993; Konttinen et al., 1994). Presynaptic afferents to the DH display increased electrophysiological activity (Guilbaud et al., 1985) and increased synthesis and release of substance P (SP), calcitonin gene-related peptide (CGRP), and glutamate (Oku et al., 1987; Kuraishi et al., 1989; Kar et al., 1991; Calza et al., 1997). These events are likely to trigger a progressive sensitization of NMDA receptors (Haley et al., 1990; Dougherty and Willis, 1992; Ren et al., 1992). As a result, postsynaptic neurons display both electrophysiological changes, which lead to dramatically increased activity (Menétrey and Besson, 1982; Calvino et al., 1987b), and long-lasting molecular modifications, which are illustrated by sustained expression of the proto-oncogene c-fos (Abbadie and Bes-

Received Nov. 10, 1998; revised April 7, 1999; accepted April 14, 1999.

This work was supported by Institut National de la Santé et de la Recherche Médicale, Association pour la Recherche contre le Cancer Grant 6995 (B.C.), and Institut UPSA de la Douleur (B.C.). S.P. is a Ministère de l'Education Nationale de la Recherche et de la Technologie fellow. We thank Dr. L. F. Reichardt for providing anti-TrkA antibodies. Drs. S. Juliano, M. Peschanski, and S. Marty are also acknowledged for helpful reading of this manuscript.

Correspondence should be addressed to Dr. B. Calvino, Institut National de la Santé et de la Recherche Médicale U421, Faculté de Médecine, 8, rue du Général Sarrail, F-94010 Créteil Cedex, France.

Copyright (C) 1999 Society for Neuroscience $\quad 0270-6474 / 99 / 195482-11 \$ 05.00 / 0$ son, 1992). These results support the existence of long-term functional adaptive changes in the first central relay of nociception during chronic pain states.

Neurotrophins, which include molecules of the nerve growth factor (NGF) family, promote the survival of sensory neurons during development and have various pharmacological effects on these neurons in adulthood (Mendell, 1996). In particular, NGF plays a crucial role in nociception (Lewin and Mendell, 1993). When injected in the periphery, NGF has potent algogenic properties in animals (Lewin et al., 1993) and man (Petty et al., 1994). Although the early component of NGF-induced hyperalgesia depends on peripheral events that lead to sensitization of nociceptors (Lewin et al., 1993), the later phase of NGF effects likely involves central NMDA receptors (Lewin et al., 1994), in correlation with electrophysiological observations described above.

Considering that neurotrophins are major determinants of plasticity linked to increased neuronal activity in the mature CNS (Gall, 1993; Thoenen, 1995), we hypothesized an involvement of NGF in chronic pain-related central plasticity. The present study aimed at investigating changes of immunoreactivity (IR) for TrkA, the high-affinity receptor of NGF, in the spinal cord of arthritic rats. Adjuvant-induced arthritis (AIA) in rats is characterized by a strong disseminated inflammation, associated with ankle hyperalgesia, and a chronic pain state (De Castro Costa et al., 1981; Calvino et al., 1987a; Colpaert, 1987). Our results show an increased number of TrkA-IR profiles at the peak of AIA, specifically in laminae V-VI of the lumbar dorsal horn.

\section{MATERIALS AND METHODS}

Animals

Experiments were performed in 43 male Sprague Dawley rats (Charles River, Saint-Aubin les Elbeuf, France), weighing 250-300 gm at the beginning of the experiment. In this rat strain, AIA is a systemic 
autoimmune disease induced by complete Freund's adjuvant (CFA) injections in both hindpaws of $50 \mu \mathrm{l}$ of CFA [6 mg of Mycobacterium butyricum (Difco Laboratory, Detroit, MI), suspended into $1 \mathrm{ml}$ of an emulsion of liquid paraffin $/ 0.9 \% \mathrm{NaCl} /$ Tween $80,6: 4: 1$ respectively, as described previously (Calvino et al., 1991)]. For injections, animals were briefly anesthetized with $4 \%$ halothane in nitrous oxide/oxygen mixture $(2: 1 \mathrm{v} / \mathrm{v})$. Control animals received $50 \mu \mathrm{l}$ of either incomplete Freund's adjuvant (IFA) or saline.

All experiments were performed in accordance with the European Communities Council Directive of November 24, 1986 (86/609/EEC). Guidelines on ethical standards for investigations of experimental pain in animals were followed (Committee for Research and Ethical Issues of the IASP, 1980). Accordingly, the number of arthritic animals was kept to a minimum. Rats were housed three to a large cage to minimize the possibility of painful interactions. They were kept at a constant room temperature of $22^{\circ} \mathrm{C}$, with a $12 \mathrm{hr}$ alternating light/dark cycle. Food and water were available ad libitum. Food was directly available on the sawdust in the cages to minimize potentially painful movements.

Quantifications of TrkA-IR neurons on longitudinal sections were performed in five arthritic rats and six control animals (IFA, $n=3$; saline, $n=3$ ).

Double-labelings of TrkA-IR-CGRP-IR and TrkA-IR-substance P-IR were analyzed in coronal sections in 18 arthritic rats and 14 control animals (IFA, $n=3$; saline, $n=11$ ). Among these animals, three arthritic and four saline-injected rats received stereotaxic injections of FluoroGold (FG) (Fluorochrome Inc., Englewood, NJ) into the right thalamus, and four arthritic and four saline-injected rats received stereotaxic injections of FG into the brainstem. All stereotaxic injections were performed 1 week before induction of arthritis. Analysis of the laminar distribution of TrkA-IR neurons in the spinal cord was performed on coronal sections of four arthritic and three saline-injected animals.

Animals were killed 4 weeks after induction of arthritis, i.e., during the acute phase of AIA.

\section{Clinical and behavioral studies}

To assess the evolution of the disease, several parameters were considered on the day the animals were killed: (1) the weight gain, measured as the difference between weights at perfusion and injection days; (2) the diameters of the ankles; and (3) a mobility score with a 5 levels scale: 4, the rat walks and runs normally; 3 , the rat runs with difficulty, but walks normally; 2 , the rat walks with difficulty; 1 , the rat crawls; 0 , the rat lies down only (Butler et al., 1985).

A pain-related test was conducted before the animals were killed. The "foot-bend" procedure evaluates the ankle hyperalgesic state (Kuzuna and Kawai, 1975; Winter et al., 1979). It involves holding the rat comfortably and gently extending the left hindpaw. The test was repeated five times at $5 \mathrm{sec}$ intervals; a rating of 1 or 0 was given if the animal emitted a squeak (1) or not (0). For each animal, the rating ranged from 0 to 5. Then, the left hindpaw was gently flexed five times at $5 \mathrm{sec}$ interval, with identical rating.

\section{Stereotaxic injections}

Seven rats received stereotaxic injections of $2 \%$ FG into the right ventrobasal complex of the thalamus, using a $1 \mu \mathrm{l}$ Hamilton microsyringe. A total of nine sites were injected $(100 \mathrm{nl} / \mathrm{site})$ at three coronal planes: anteroposterior (AP), +5.2, +5.8, and +6.7; height $(\mathrm{H}),+4.0$; and lateral (L), 1, 2, and $3 \mathrm{~mm}$ for each plane (Paxinos and Watson, 1986).

Eight rats received one stereotaxic injection of $100 \mathrm{nl}$ of $2 \% \mathrm{FG}$ into the brainstem with the following coordinates: AP, -4.5 ; L, 1.9; and $\mathrm{H}, 0$.

\section{Immunohistochemistry}

Animals were deeply anesthetized with sodium pentobarbitone $(50 \mathrm{mg} /$ $\mathrm{kg}$, i.p.) and were perfused intracardially with $100 \mathrm{ml}$ of $0.1 \mathrm{M}$ Tris buffer, $\mathrm{pH} 7.4$, containing $0.9 \% \mathrm{NaCl}$, followed by $500 \mathrm{ml}$ of $4 \%$ paraformaldehyde and $15 \%$ of a saturated solution of picric acid, in $0.1 \mathrm{M}$ phosphate buffer (PB), pH 7.4. The lumbar spinal cord was removed on ice, post-fixed in the same fixative for $12 \mathrm{hr}$, and cryoprotected in $30 \%$ sucrose in PB. Lumbar frontal sections of $30 \mu \mathrm{m}$ were cut with a cryostat and were serially collected in $\mathrm{PB}$ containing $0.9 \% \mathrm{NaCl}$ (PBS), to be processed for immunohistochemistry.

For peroxidase immunohistochemistry, tissue sections were incubated with primary antibodies in PBS containing 0.3\% Triton X-100 (PBST) for $48 \mathrm{hr}$ at $+4^{\circ} \mathrm{C}$. Primary antibodies included rabbit anti-TrkA ectodomain (Clary et al., 1994; 1:4000), rabbit anti-CGRP (1:15000; Peninsula Laboratories, Belmont, CA), and rabbit anti-substance P (1:4000; Pen- insula Laboratories). After three washes in PBST, the sections were incubated for $1 \mathrm{hr}$ in 1:400 Elite streptavidin-biotin-peroxidase complex (Vector Laboratories, Burlingame, CA). Sections were washed three times in PBS, and peroxidase activity was revealed by $3,3^{\prime}-$ diaminobenzidine (DAB) (Vector Laboratories). For double CGRPTrkA and SP-TrkA immunohistochemistry, tissue sections were incu-

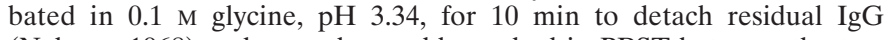
(Nakane, 1968) and were thoroughly washed in PBST between the two immunohistochemical procedures. CGRP and substance P immunohistochemistry were performed according to the protocol described above, but peroxidase activity was revealed by the "SG" compound of Vector Laboratories. Tissue sections were then washed in PBS and mounted on gelatin-coated slides, dehydrated, and coverslipped in Permount.

For immunofluorescence, sections were incubated $48 \mathrm{hr}$ at $+4^{\circ} \mathrm{C}$ in anti-TrkA primary antibodies $(1: 2000)$. After washing, they were incubated with the secondary antibody (anti-rabbit Cy3-labeled; 1:1000; Jackson ImmunoResearch, West Grove, PA). Sections were washed, mounted on gelatin-coated slides, air dried, and coverslipped with Vectashield (Vector Laboratories). Sections were examined using a Zeiss (Oberkochen, Germany) Axophot fluorescence microscope, with the following emission wavelengths: UV filter, $420 \mathrm{~nm}$; green filter, $590 \mathrm{~nm}$.

The specificity of primary antibodies was tested by the omission of the primary or secondary antibodies. Preadsorption with the corresponding synthetic peptides have been described previously (Clary et al., 1994; Michael et al., 1997). The absence of fluorescence cross talk within the two fluorophores Cy3 and FG was verified by the lack of Cy3 signal using the UV filter and, conversely, the lack of FG fluorescence using the green filter.

\section{Quantifications}

In all these quantifications, the same spinal cord level was dissected out and analyzed in arthritic and control animals (using vertebral marks). Sections were randomly sampled, taken at regular intervals from a randomly starting point.

Quantification of TrkA-IR profiles on longitudinal sections. After perfusion, every lumbar spinal cord was sectioned at the dorsal root ganglion level of the 11th thoracic and the 2nd lumbar segments (spinal cord levels: L1 to S3). Every fourth horizontal longitudinal section (30 $\mu \mathrm{m}$ thickness) from the dorsal surface of the spinal cord to the central canal was cut with a cryostat, pooled, and processed for TrkA immunohistochemistry.

The total number of TrkA-IR profiles counted in all sections per animal was calculated. For this quantification study and the following, profiles were considered positive when they were clearly labeled. Comparisons between the mean values of control and arthritic rats were performed by statistical analysis using one-way ANOVA.

Laminar distribution of TrkA-IR profiles. In every 10th coronal section of the lumbar enlargement (L2-L5), the number of TrkA-IR profiles per lamina was determined in four arthritic and three control animals. Results are expressed as the mean \pm SEM of TrkA-IR profiles in laminae V-VI, VII, and X, for each section. Statistical analysis was performed using nonparametric Mann-Whitney $U$ test.

Quantification of TrkA-IR spinothalamic and spinoreticular neurons. The number of TrkA-labeled, FG-labeled, and double-labeled TrkA-IR-FG profiles was counted in every 10th coronal section of the lumbar enlargement, contralateral to FG injection sites. Among the whole population of FG-labeled neurons, the percentage of double-labeled neurons projecting either to the ventrobasal complex of the thalamus or to the reticular nucleus of the brainstem (so-called "spinothalamic" or "spinoreticular" TrkA-IR neurons, respectively) was calculated as the ratio of the number of profiles double-labeled, divided by the total number of FG-labeled profiles, multiplied by 100 .

\section{RESULTS}

No clinical or behavioral changes were observed in control animals injected with saline or IFA. Arthritic rats displayed a strong reduction of weight gain $(-27.3 \pm 7.6$ in arthritic rats; $+142.3 \pm$ 17.7 in IFA-injected rats; $+123 \pm 7.0$ in saline-injected animals), difficulties in walking (mobility score: $1.6 \pm 0.2$ in arthritic rats vs $4.0 \pm 0$ in both groups of control animals), and increased ankle diameters $(5.2 \pm 0.2 \mathrm{~mm}$ in arthritic rats vs $2.79 \pm 0.04 \mathrm{~mm}$ in saline-injected rats and $2.78 \pm 0.06 \mathrm{~mm}$ in IFA-injected rats). The foot-bend procedure showed an ankle hyperalgesia in arthritic animals (flexion and extension scores: $4.1 \pm 0.3$ and $0.4 \pm 0.2$, 

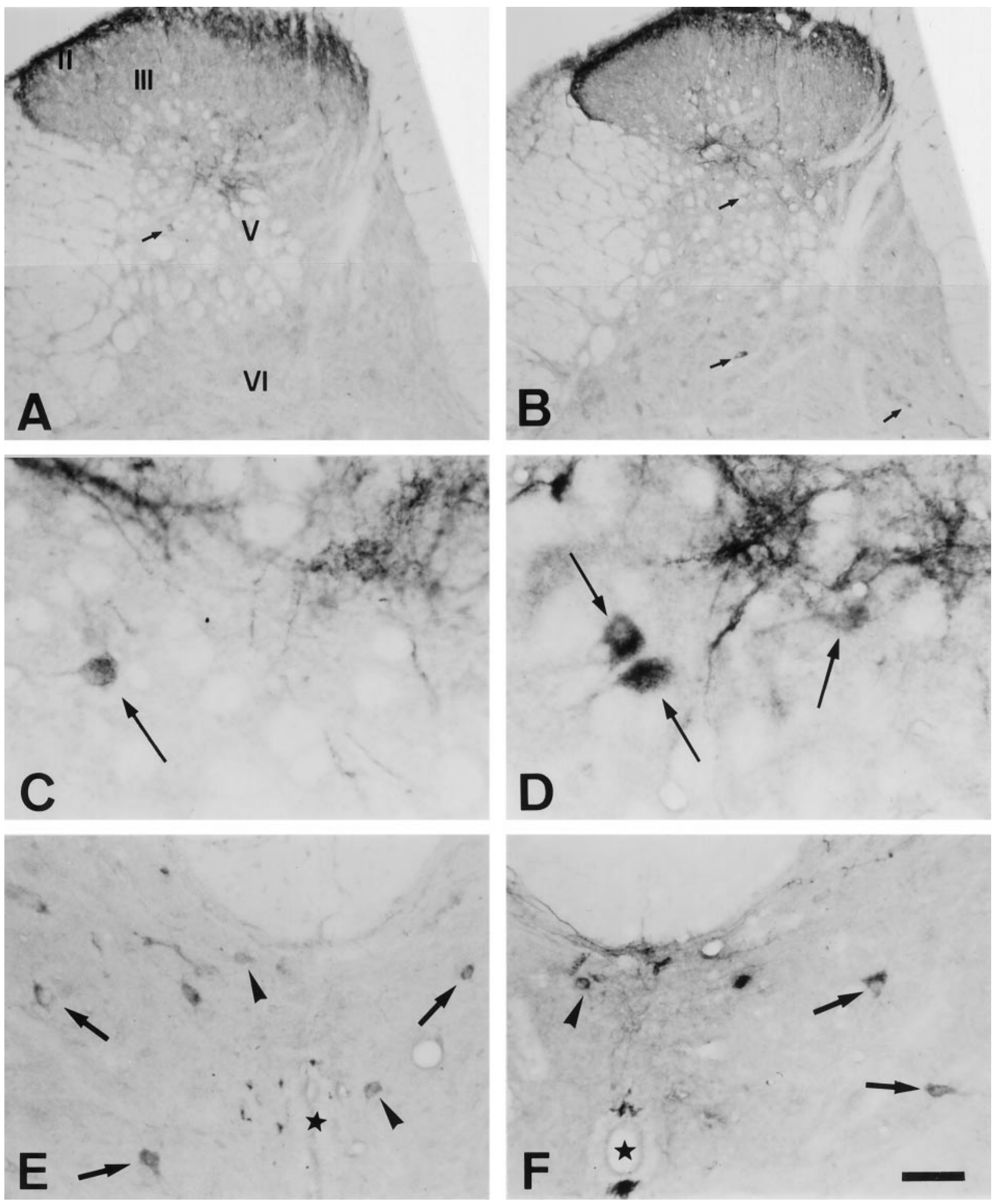

Figure 1. Photomicrograph showing TrkA immunoreactivity in the spinal cord of a control animal injected with saline $(A, C, E)$ and in an arthritic rat $(B, D, F)$ during the acute phase of AIA. TrkA-IR fibers are present in laminae I-V $(A, B)$, whereas immunoreactive neuronal profiles are located in laminae V-VI $(A-D$, arrows $), \mathrm{VII}(E, F$, arrows), and X $(E, F$, arrowheads). $C$ and $D$ are high-power photomicrographs of the reticular part of lamina $\mathrm{V}$ showing both TrkA-IR terminal fibers and increased number of TrkA-IR neurons (arrows) in arthritic rats $(D)$ versus control animals $(C)$. An increased number of TrkA-IR neurons is observed in lamina V $(A-D)$, whereas no changes are observed in laminae V II and X $(E, F)$. Stars indicate the lumen of the central canal. Scale bar: $A, B, 120 \mu \mathrm{m} ; C, D, 20 \mu \mathrm{m} ; E, F, 60 \mu \mathrm{m}$. 

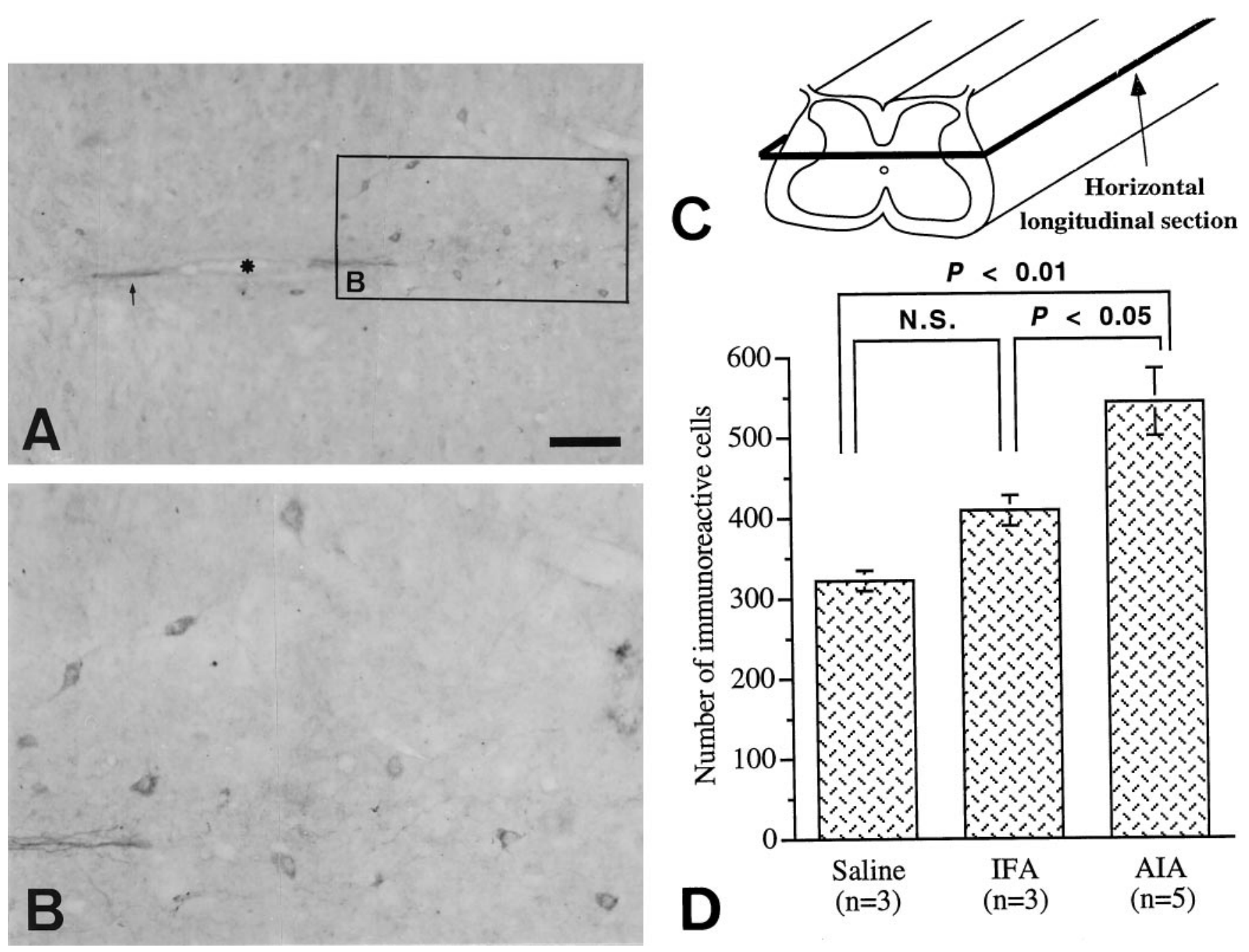

Figure 2. Quantification of TrkA-IR profiles in horizontal longitudinal sections of the lumbar dorsal horn in AIA. $A, B$, Photomicrograph showing TrkA-IR neurons in a longitudinal section of the DH in one arthritic rat. Asterisk in $A$ indicates the central canal; arrow points to TrkA-IR axonal profiles. Note the density and multipolar shape of TrkA-IR neurons $(B)$. $C$, Schematic representation of horizontal longitudinal section in the spinal cord. $D$, Countings of TrkA-IR neuronal profiles in the DH of control animals injected with either saline or IFA and in AIA rats during the acute phase of the disease (4 weeks after injection). Results are expressed as the mean \pm SEM total number of TrkA-IR neuronal profiles counted in every fourth section. Statistical analysis was performed using one-way ANOVA. The level of significance was set as $* p<0.05$ and $* * p<0.01$. NS, Nonsignificant difference. Scale bar: $A, 130 \mu \mathrm{m} ; B, 60 \mu \mathrm{m}$.

respectively, in arthritic vs $0 \pm 0$ and $0 \pm 0$, respectively, in both control groups).

\section{TrkA immunoreactivity in the spinal cord of control and arthritic animals}

In both arthritic and control animals, TrkA immunostaining was present in terminals and varicose fibers in superficial laminae (I-II) of the DH. TrkA-IR fibers radiated through lamina III to ramify in a dense plexus in laminae IV-V (Fig. 1A,B). A few thin fibers were seen in the medial part and in the lateral reticular part of lamina V. TrkA-IR neurons were observed in laminae V, VI, VII, and X. In laminae V and VI, IR cells were of large size $(20-30 \mu \mathrm{m})$ and multipolar. They were observed in both the medial and the lateral reticular part of lamina $\mathrm{V}$ (Fig. 1C,D). TrkA-IR cells observed in lamina VII were of large size (20-26 $\mu \mathrm{m})$ and multipolar or spindle-shaped (Fig. $1 E, F$ ). In lamina X, TrkA-IR cells had a round shape, a small size $(10 \mu \mathrm{m})$, and were located in the dorsal part of the central canal (Fig. 1E,F). The distribution pattern and morphology of TrkA-IR neurons was the same in arthritic and control animals. Quantification of TrkA-IR profiles in the lumbar spinal cord (Fig. 2) showed a statistically significant increase in arthritic rats when compared with control animals injected with saline or IFA. The difference observed in IFA-injected animals when compared with saline-injected animals was not significant. Quantification of the laminar distribution showed that this increase concerned specifically profiles located in laminae V-V I, mainly at L3 and L4 lumbar spinal cord levels (Fig. 3). In L2 and L5, differences were statistically nonsignificant. No difference was seen in the mean number of TrkA-IR profiles in laminae VII and X in the four lumbar levels for both groups of animals.

\section{Double-labelings of FG-labeled neurons and TrkA immunohistochemistry}

Spread of the tracer resulting from injections of FG into the thalamus was closely similar in all experiments, and one representative example is given in Figure $4 A$. Retrogradely FG-labeled spinothalamic neurons were present in laminae I-II (data not shown), V-VI, VII, and X, the main part being located in lamina VII (Fig. 5A). Observations on a fluorescent microscope showed 


\section{Saline}
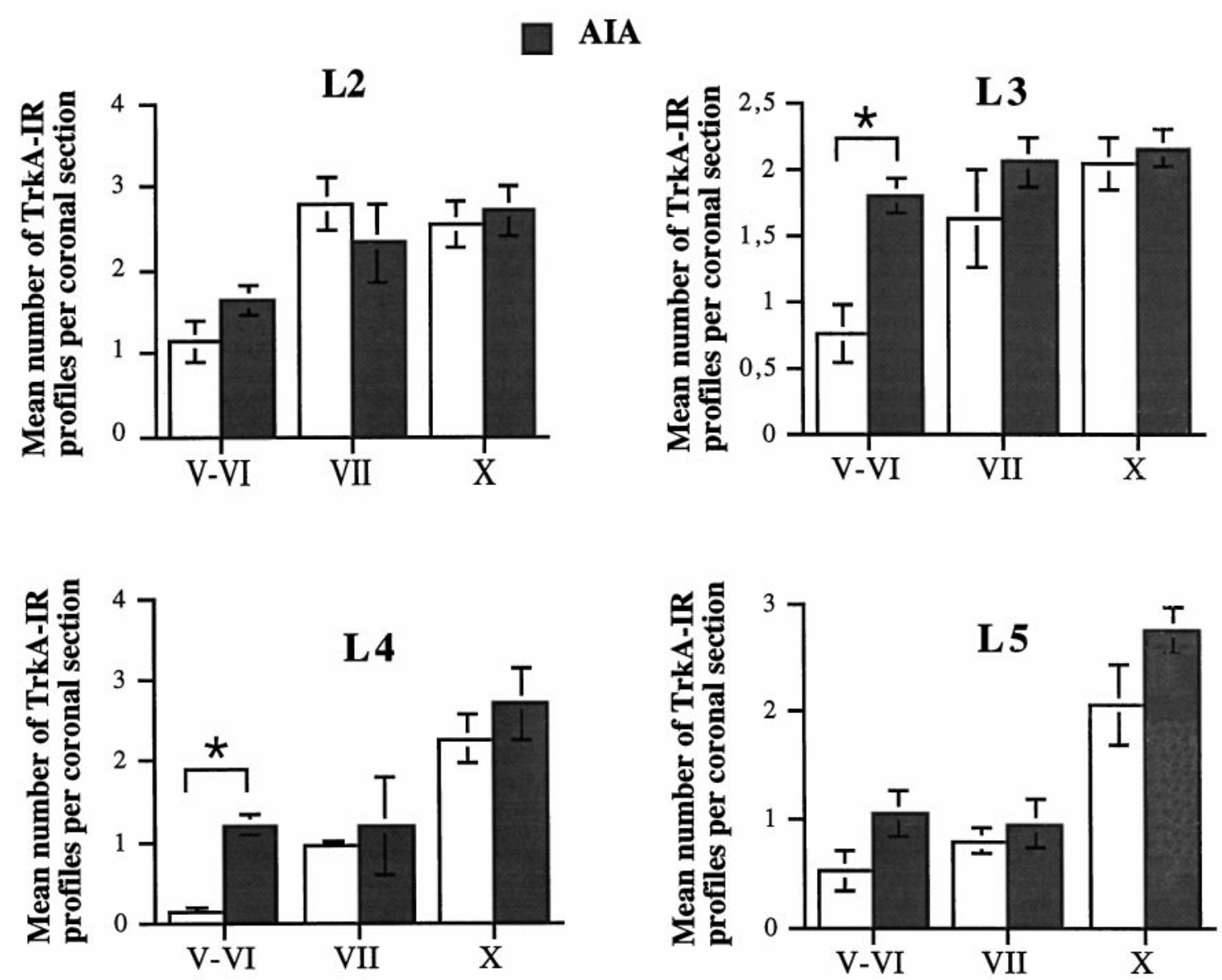

Figure 3. Laminar distribution of TrkA-IR profiles in the lumbar spinal cord at four lumbar levels (L2-L5) in control $(n=3)$ and arthritic $(n=4)$ rats at 4 weeks after injection in AIA. Results are expressed as the mean \pm SEM of immunoreactive profiles for each region and per coronal section. Statistical analysis was performed using Mann-Whitney $U$ nonparametric test. The level of significance was set as *p $p 0.05$.

that part of TrkA-IR neurons in laminae V, VI, VII, and X were FG-labeled (Fig. 5C,E). Quantifications showed no differences in the mean number of FG-traced neurons in both groups of animals. Countings of fluorescent TrkA-IR profiles revealed a significantly increased number of TrkA-IR profiles in laminae V-V I of arthritic rats at lumbar levels L3 and L4 (Table 1), as described in Figure 3 with a DAB revelation in an another set of experiments. Approximately 5-10\% of the total number of FG-labeled profiles were double-labeled, i.e., TrkA-IR, in both control and arthritic rats (Table 2). No significant differences in percentage of colocalization could be observed between both groups of animals for each lamina and at all lumbar levels examined (Table 2).

Injections of FG into the brainstem resulted in labeling of the lateral reticular nucleus, the nucleus cuneatus and gracilis, part of the nucleus of the solitary tract, and part of the parvocellular reticular nucleus (Fig. 4B). In the spinal cord, retrogradely FGlabeled neurons were mainly located in laminae V-VI but also in laminae VII and X (Fig. 5B) and in lamina I to a lesser extent. The mean number of FG-traced spinoreticular neurons was not different in control and arthritic rats (Table 1). However, as described above after retrograde tracing from the thalamus, countings of fluorescent TrkA-IR profiles showed a significantly increased number of TrkA-IR profiles in laminae V-VI of arthritic rats at lumbar levels L3-L4 (Table 1), as described in
Figure 3. Quantifications showed that a part of FG-labeled spinoreticular profiles in laminae V-VI (Table 1), VII, and X were TrkA-IR in both control and arthritic animals, and the percentage of these profiles double-labeled increased significantly in arthritic rats at lumbar levels L3-L4 in laminae V-VI (Table 2, Fig. $5 D, F)$. Laminar distribution analysis showed that this significant increase was not observed in laminae VII and X of the dorsal horn (Table 2). No significant differences coud be evidenced at lumbar levels L2 and L5 (Table 2).

These results show that, at L3-L4 levels, $\sim 10 \%$ of spinothalamic neurons were TrkA-IR in both control and arthritic rats. Conversely, whereas a very few spinoreticular neurons were TrkA-IR in control animals, in arthritic rats $22.8 \%$ at L3 and $19.7 \%$ at L4 levels of these spinoreticular neurons express TrkA.

\section{TrkA-CGRP and TrkA-substance $P$ double-labelings}

CGRP-IR fibers were observed in superficial laminae of the spinal cord of control and arthritic rats. Straight fibers penetrated lamina III of the dorsal horn and ramified at the interface of laminae IV-V. Some were seen running through lamina V (Fig. $6 A)$. Numerous fibers running down from layers $\mathrm{V}-\mathrm{VI}$ along the medial aspect of the gray matter reached lamina $\mathrm{X}$ (Fig. $6 C$ ). In addition, few fibers were observed in laminae VII-V III of the ventral horn. CGRP-IR fibers were increased in layers IV-V in 
A
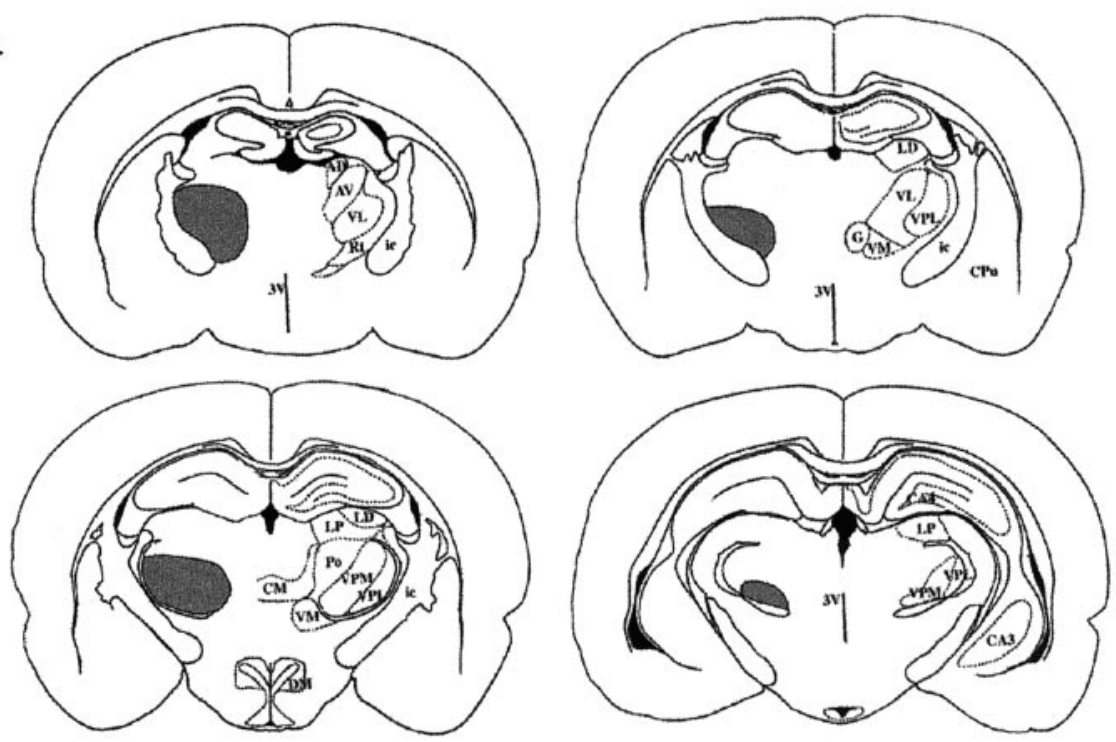

B

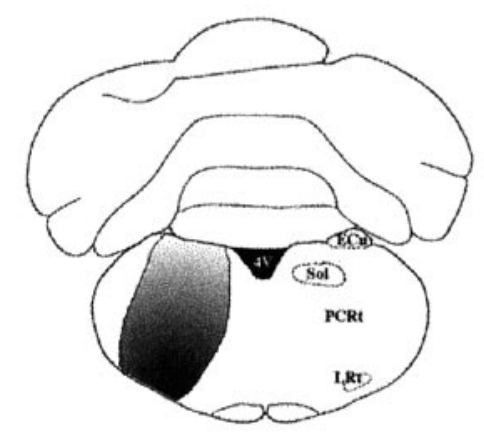

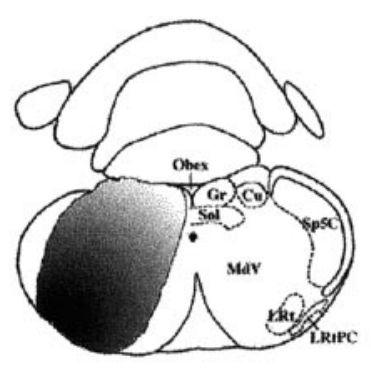

Figure 4. Drawings showing the spread of FG injection in the right thalamus [from rostral (left) to caudal $(r i g h t)](A)$ and in the brainstem $(B)$. Areas traced were the ventrobasal complex of the thalamus, part of the nucleus of the solitary tract, and the reticular nucleus (medullatory and lateral parts). $3 V$, Third ventricle; $4 V$, fourth ventricle; $A D$, anterodorsal thalamic nucleus; $A V$, anteroventral thalamic nucleus; $C A 3$, CA3 field of Ammon's horn; CA4, CA4 field of Ammon's horn; $C M$, central medial thalamic nucleus; $C p u$, caudate putamen; $C u$, cuneate nucleus; $D M$, dorsomedial hypothalamic nucleus; $E C u$, external cuneate nucleus; $G$, nucleus gelatinosus of the thalamus; $i c$, internal capsule; $L D$, laterodorsal thalamic nucleus; $L P$, lateroposterior thalamic nucleus; $L R t$, lateral reticular nucleus; $L R t P C$, lateral reticular nucleus, parvocellular; $M d V$, medullatory reticular nucleus, ventral part; $P C R t$, parvocellular reticular nucleus; $P o$, posterior thalamic nuclear group; Rt; reticular thalamic nucleus; Sol, nucleus of the solitary tract; $S p 5$, spinal trigeminal nucleus; $V L$, ventrolateral thalamic nucleus; $V M$, ventromedial thalamic nucleus; $V P L$, ventroposterior thalamic nucleus, lateral part; $V P M$, ventroposterior thalamic nucleus, medial part. arthritic rats, as described previously. Double-labelings of TrkA and CGRP revealed that TrkA-IR neurons in laminae $\mathrm{V}$ and $\mathrm{X}$ were present mainly in projection fields of CGRP-IR fibers (Fig. $6 A-C)$. In lamina $\mathrm{V}$, TrkA-IR neurons were surrounded by dense plexuses of CGRP-IR fibers, with axonal varicosities and intervaricose segments disposed in close contact with their soma and dendrites (Fig. 6B).

Substance P immunoreactivity labeled thin and abundant varicosities in superficial and deep laminae of the dorsal horn (I, II, $\mathrm{V}$ ) and around the central canal in lamina X (Fig. 6E) of control and arthritic rats. A weaker density was observed in the medial lamina VII (Fig. 6F). In all these layers, TrkA-IR neurons were closely surrounded by a dense plexus of immunoreactive fibers (Fig. 6D-F).

\section{DISCUSSION}

Our study describes an increased number of TrkA-IR profiles in the dorsal spinal cord of rats suffering from AIA. Increases occurred in laminae V-VI in the projection fields of CGRP-IR and SP-IR afferent fibers, laminae in which long-term electrophysiological and molecular changes have been described during the course of AIA. Investigations using retrograde tract tracing and double-label immunohistochemistry showed that TrkA-IR profiles belonged to spinothalamic and spinoreticular pathways. However, TrkA-IR was increased only in spinoreticular neurons at lumbar levels L3 and L4, which are somatotopic projection sites of hindlimbs and ankles in which inflammation is maximal at 3 weeks after injection. These results suggest a specific increase in the number of TrkA receptors in the first central relay of nociception, supporting the hypothesis that NGF might be involved in neuronal plasticity linked to chronic pain.

\section{TrkA-IR neurons and AIA}

In agreement with previous descriptions in rats (Sobreviela et al., 1994; Averill et al., 1995; Molliver et al., 1995; Michael et al., 1997), both axonal and somatic localizations of TrkA-IR were observed in the spinal DH. Three neuronal populations containing TrkA protein and mRNA have been described in the spinal cord according to their location, size, and neurochemical characteristics. Small neurons located in the dorsal part of the central canal (lamina X) correspond mainly to cholinergic propriospinal neurons. The medial part of lamina VII contains multipolar cholinergic, propriospinal partition cells also described as TrkAIR. Neurons located in laminae VII and $\mathrm{X}$ are involved in integration of articular and visceroceptive information, respectively (Menétrey et al., 1984a,b; Ness and Gebhart, 1990). Laminae V and VI contain noncholinergic, nonpropriospinal, large multipolar neurons that are also TrkA-IR (Michael et al., 1997). Although labeled neurons displayed similar distribution patterns and morphology in control animals and arthritic rats, an increased number of TrkA-IR profiles was observed specifically in laminae $\mathrm{V}-\mathrm{VI}$ in animals with AIA. No changes were observed in other DH laminae. 

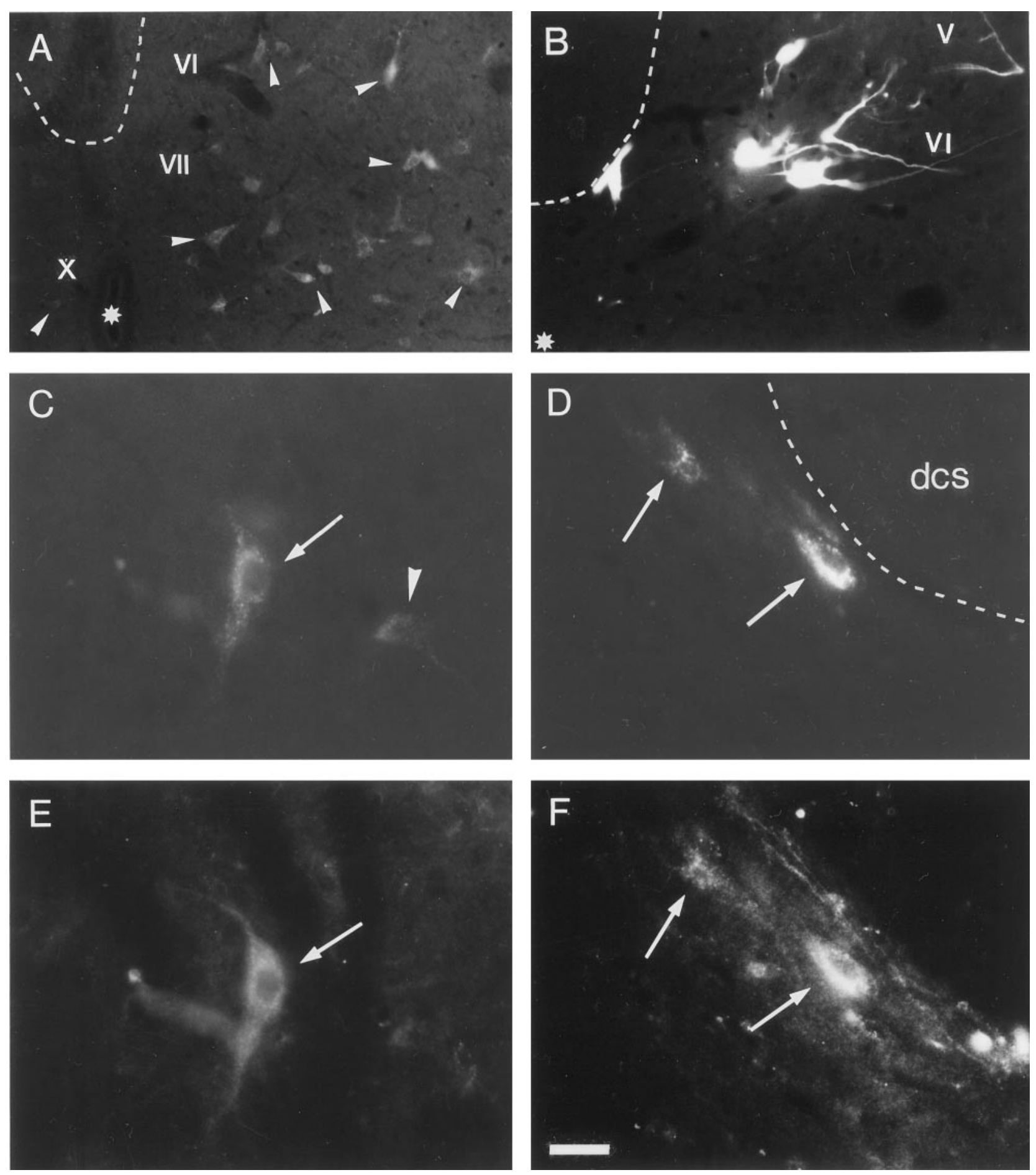

Figure 5. Photomicrographs showing FG spinothalamic $(A, C, E)$ and spinoreticular $(B, D, F)$ traced neurons and the colocalization with TrkA $(E, F)$ in the lumbar dorsal horn in representative arthritic rats. $A, B$, Laminar distribution of FG-traced neurons. Spinothalamic neurons are located in laminae V, VI, VII, and X (A, arrowheads), whereas spinoreticular neurons are mainly located in laminae V-VI $(B)$. $C-F$, Partial colocalization of TrkA-IR (E, $F$, arrows) in FG-traced spinothalamic ( $C$, arrow, arrowhead) and spinoreticular ( $D$, arrows) neurons. One FG-traced spinothalamic neuron not TrkA-IR is shown in $C$ (arrowhead). Asterisks indicate the central canal, and dotted lines in $A, B$, and $D$ indicate the outline of the dorsal corticospinal tract (dcs). Scale bar: $A, 80 \mu \mathrm{m} ; B, 40 \mu \mathrm{m} ; C-F, 25 \mu \mathrm{m}$.

Neurons in laminae V-VI have been classified chiefly as nonspecific nociceptive, or wide dynamic range (WDR), neurons. They receive both nociceptive and non-nociceptive input from primary sensory neurons, as well as local and descending modulatory inputs (Besson and Chaouch, 1987). Central sensitization, expressed as increased excitability and activity of deep DH neurons, and plasticity of receptive fields (Cook et al., 1987) is fundamental to the genesis of inflammatory chronic pain (Woolf and McMahon, 1985). The WDR neurons of rats suffering from arthritis display expansion of their receptive fields, accompanied 
Table 1. FG-labeled profiles in control and arthritic rats

\begin{tabular}{|c|c|c|c|c|c|c|c|c|}
\hline & \multicolumn{2}{|l|}{ L2 } & \multicolumn{2}{|l|}{ L3 } & \multicolumn{2}{|l|}{ L4 } & \multicolumn{2}{|l|}{ L5 } \\
\hline & Saline & AIA & Saline & AIA & Saline & AIA & Saline & AIA \\
\hline \multicolumn{9}{|c|}{ Spinoreticular neurons } \\
\hline FG & $19.3 \pm 4.1$ & $18.3 \pm 3.9$ & $18.0 \pm 1.8$ & $24.3 \pm 4.3$ & $12.5 \pm 2.2$ & $16.8 \pm 5.3$ & $9.75 \pm 1.3$ & $13.0 \pm 4.24$ \\
\hline TrkA-IR & $6.7 \pm 0.9$ & $8.0 \pm 2.4$ & $2.5 \pm 0.3$ & $10.5 \pm 3.0^{*}$ & $1.8 \pm 0.5$ & $6.8 \pm 0.8^{*}$ & $0.8 \pm 0.5$ & $4.5 \pm 1.7$ \\
\hline Double-labeled & $2.25 \pm 0.85$ & $5.25 \pm 1.97$ & $0.25 \pm 0.25$ & $6.50 \pm 3.30 *$ & $0.0 \pm 0.0$ & $3.25 \pm 1.49 *$ & $0.0 \pm 0.0$ & $1.25 \pm 0.95$ \\
\hline \multicolumn{9}{|c|}{ Spinothalamic neurons } \\
\hline FG & $10.8 \pm 1.4$ & $7.5 \pm 0.4$ & $20.0 \pm 3.1$ & $13.3 \pm 2.3$ & $19.8 \pm 5.1$ & $11.7 \pm 1.8$ & $11.8 \pm 0.9$ & $8.7 \pm 3.0$ \\
\hline TrkA-IR & $3.3 \pm 0.5$ & $3.5 \pm 0.4$ & $2.8 \pm 0.3$ & $7.0 \pm 3.0 *$ & $2.5 \pm 0.3$ & $4.0 \pm 0.0^{*}$ & $0.75 \pm 0.5$ & $0.7 \pm 0.7$ \\
\hline Double-labeled & $0.50 \pm 0.29$ & $0.5 \pm 0.35$ & $1.25 \pm 0.63$ & $1.33 \pm 0.76$ & $1.00 \pm 0.40$ & $0.33 \pm 0.33$ & $0.0 \pm 0.0$ & $0.33 \pm 1.73$ \\
\hline
\end{tabular}

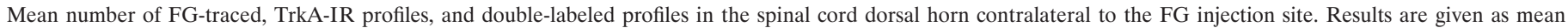
number \pm SEM in five coronal sections. Statistical analysis was performed using Mann-Whitney $U$ nonparametric test. ${ }^{*} p<0.05$.

Table 2. FG-labeled profiles in control and arthritic rats

\begin{tabular}{|c|c|c|c|c|c|c|}
\hline & \multicolumn{2}{|c|}{ Laminae V-VI } & \multicolumn{2}{|c|}{ Lamina VII } & \multicolumn{2}{|l|}{ Lamina $\mathrm{X}$} \\
\hline & Saline & AIA & Saline & AIA & Saline & AIA \\
\hline \multicolumn{7}{|c|}{ Spinoreticular neurons } \\
\hline $\mathrm{L} 2$ & $10.5 \pm 3.6$ & $28.0 \pm 6.2$ & $3.1 \pm 3.1$ & $7.2 \pm 7.2$ & $0.0 \pm 0.0$ & $0.0 \pm 0.0$ \\
\hline L3 & $1.7 \pm 1.7$ & $22.8 \pm 3.4^{*}$ & $0.0 \pm 0.0$ & $6.1 \pm 3.6$ & $0.0 \pm 0.0$ & $0.0 \pm 0.0$ \\
\hline L4 & $0.0 \pm 0.0$ & $19.7 \pm 7.0^{*}$ & $0.0 \pm 0.0$ & $2.1 \pm 2.1$ & $0.0 \pm 0.0$ & $0.0 \pm 0.0$ \\
\hline L5 & $0.0 \pm 0.0$ & $18.1 \pm 10.7$ & $0.0 \pm 0.0$ & $0.0 \pm 0.0$ & $0.0 \pm 0.0$ & $0.0 \pm 0.0$ \\
\hline \multicolumn{7}{|c|}{ Spinothalamic neurons } \\
\hline $\mathrm{L} 2$ & $4.4 \pm 2.6$ & $7.2 \pm 4.5$ & $10.1 \pm 4.2$ & $9.4 \pm 5.9$ & $12.2 \pm 7.2$ & $50.0 \pm 31.6$ \\
\hline L3 & $6.9 \pm 3.1$ & $9.0 \pm 5.0$ & $9.9 \pm 5.9$ & $4.3 \pm 1.8$ & $0.0 \pm 0.0$ & $0.0 \pm 0.0$ \\
\hline L4 & $11.9 \pm 4.8$ & $5.2 \pm 2.2$ & $8.8 \pm 5.3$ & $3.9 \pm 1.8$ & $0.0 \pm 0.0$ & $0.0 \pm 0.0$ \\
\hline L5 & $0.0 \pm 0.0$ & $2.4 \pm 1.8$ & $1.8 \pm 1.8$ & $5.3 \pm 2.2$ & $0.0 \pm 0.0$ & $0.0 \pm 0.0$ \\
\hline
\end{tabular}

Mean percentage of colocalization of FG-TrkA labelings in neuronal profiles in the spinal horn of control and arthritic rats. Percentages of colocalization were calculated as the ratio of the number of double-labeled profiles divided by the total number of FG-labeled profiles in laminae V-VI of the lumbar spinal cord. Results are given as mean percentage \pm SEM. Statistical analysis was performed using Mann-Whitney $U$ nonparametric test. ${ }^{*} p<0.05$.

by dramatic electrophysiological activity (Calvino et al., 1987b) and long-lasting molecular adaptive changes (Abbadie et al., 1992). The specific increase in TrkA-IR in the singular population of laminae V-VI neurons supports our hypothesis that TrkA receptors are involved in central remodeling generated by chronic pain. In addition, increases in TrkA were strictly observed in lumbar segments L3-L4, which are the somatotopic projection sites of hindlimbs and ankles (Swett and Woolf, 1985; Molander and Grant, 1986) in which the lesions are essentially observed at 3 weeks PI in AIA (Calvino et al., 1987a).

Nonspecific nociceptive neurons of the deep layers of the $\mathrm{DH}$ receive nociceptive $(\mathrm{C}$ and $\mathrm{A} \delta$ ) and non-nociceptive $(\mathrm{A} \alpha, \mathrm{A} \beta)$ afferent fibers (Besson and Chaouch, 1987). Small-sized C and A $\delta$ fibers are mainly SP-IR and CGRP-IR (McCarthy and Lawson, 1989, 1990). In our study, axonal arborizations of CGRP-IR and SP-IR fibers were observed in close contact with TrkA-IR neurons of laminae V-VI. Only electron microscopic analysis can demonstrate a synaptic link between these elements. However, the close proximity of both systems, together with the structural features and location of TrkA-IR neurons, suggest that they belong to the first central relay of nociception.

WDR neurons have an integrative function. They convey the resulting information to the ventrobasal complex of the thalamus and to the brainstem (Jessell and Kelly, 1991). Quantification of combined tract tracing and immunohistochemistry revealed that TrkA was expressed by both spinoreticular and spinothalamic neurons in control animals. However, the increased number of
TrkA-IR profiles observed in arthritic rats paralleled an increased number of double-labeled spinoreticular neurons, whereas there was no significant changes in the number of double-labeled spinothalamic neurons. This suggests that increased number of TrkA-IR neurons in AIA involved the spinoreticular, but not the spinothalamic, pathway. This specificity shows that arthritis triggers a peculiar activation of one of the spinal ascending pathways that convey peripheral messages to upper nervous centers, thus modifying their respective functional importance. This agrees with electrophysiological data showing that the spinoreticular, rather than the spinothalamic, tract conveys nociceptive messages to supraspinal sites in arthritic rats (Guilbaud et al., 1986). The results presented here provide additional evidence that adaptive functional plasticity associated with chronic pain syndromes results, at least in part, from changes in the spinoreticular pathway.

\section{Possible involvement of NGF in chronic pain-linked central plasticity}

What is the origin of the increased number of TrkA-IR neurons in AIA? A first answer to this question could be an action of NGF as a trigger to TrkA upregulation. Indeed, in vivo, the only one triggering factor for TrkA synthesis is its main ligand. Whereas a single in vitro study evidenced that increased electrophysiological activity is able to induce TrkA synthesis without NGF (Ennulat and Stach, 1987), such a result has never been found in vivo. We then propose that this increased TrkA-IR is a result of increased NGF synthesis. 

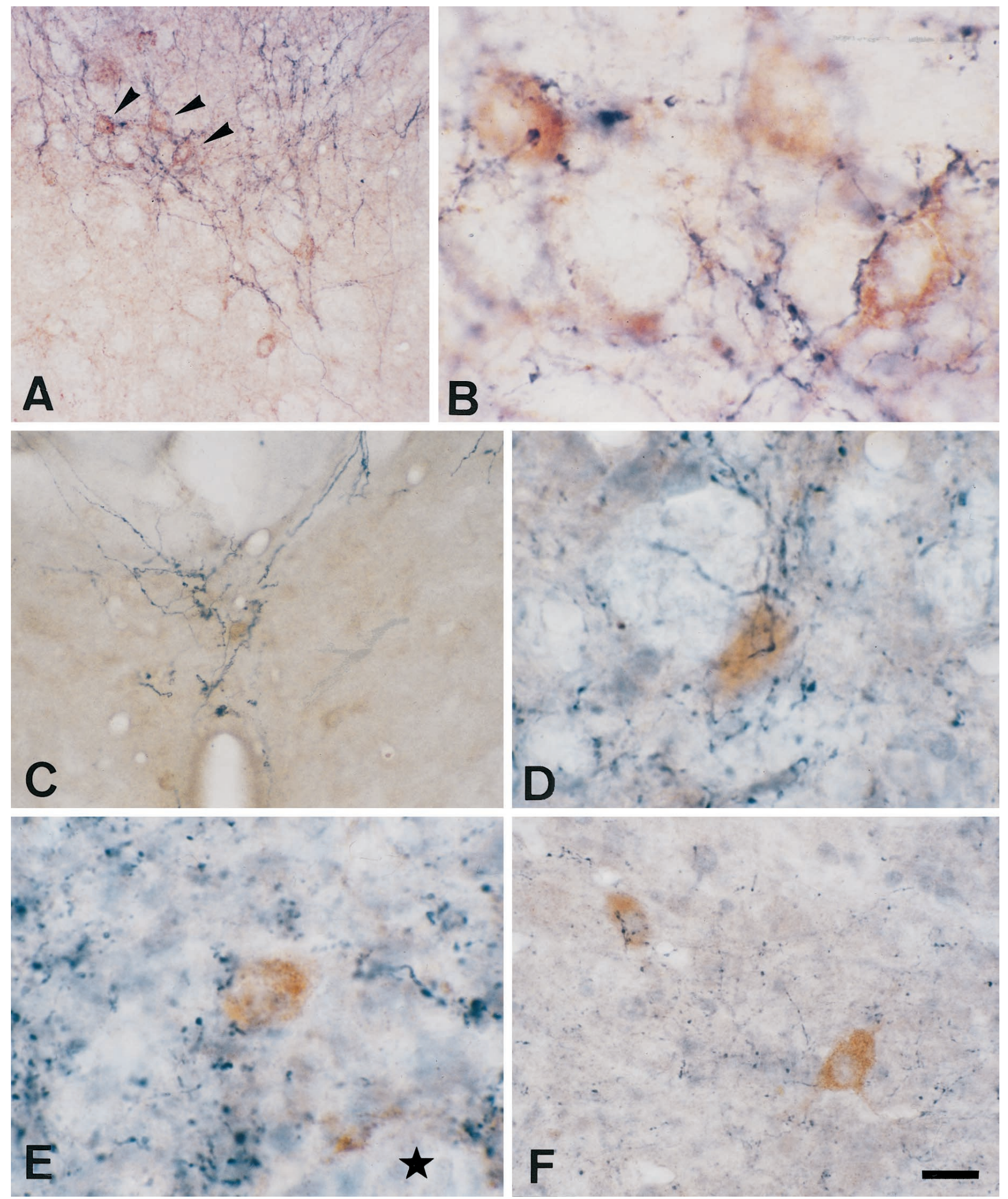

Figure 6. Double-labelings of TrkA (brown) and CGRP (blue) $(A-C)$, and TrkA (brown) and SP (blue) $(D-F)$, in layers V $(A, B, D), \mathrm{X}(C, E)$, and VII $(F)$ of the lumbar dorsal horn of arthritic rats. CGRP-IR and SP-IR fibers are located in close proximity of, and apposition to, TrkA-IR neurons (arrowhead in $A$, higher magnification in $B$ ). Star in $E$ indicates the top of the central canal. Scale bar: $A, 45 \mu \mathrm{m} ; B, 10 \mu \mathrm{m}$; $C, 60 \mu \mathrm{m} ; D, E, 8 \mu \mathrm{m}$; $F, 20 \mu \mathrm{m}$. 
The development and maintenance of inflammatory conditions in various rat disease models (Aloe et al., 1992b; Donnerer et al., 1992; Safieh-Garabadian et al., 1995) and in human arthritis (Aloe et al., 1992a) are associated with increased peripheral concentrations of NGF that is retrogradely transported from peripheral tissues to dorsal root ganglia. A central release of NGF after anterograde transport has been described in the optic tract (Von Bartheld et al., 1996), but, in contrast to its cognate molecule brain-derived neurotrophic factor, which is anterogradely transported from the DRG to the spinal cord (Tonra et al., 1998), NGF is not transported anterogradely in sensory neurons (Tonra et al., 1998). We may thus exclude the possibility of a transganglionic transport of NGF and a resulting increase in TrkA-IR in the spinal dorsal horn of arthritic rats.

In vivo, increased electrophysiological activity is associated with upregulation of NGF (Ernfors et al., 1991). Because NGF has been demonstrated to induce upregulation of TrkA mRNA by both in vitro (Lindsay et al., 1990; Wyatt and Davies, 1993) and in vivo studies (Holtzman et al., 1992; Gibbs and Pfaff, 1994), we can suggest that the increased number of TrkA-IR profiles observed in this study could be a result of NGF synthesis induced by increased electrophysiological activity of nociceptive pathways. NGF synthesis can thus be upregulated at spinal levels and/or within targets of the spinoreticular pathway. In the later case, NGF would be retrogradely transported from the reticular nuclei to the spinal cord, inducing TrkA synthesis. This hypothesis is currently under investigation.

In conclusion, our study shows that central functional adaptive changes set up during the development of AIA in parallel with changes in pain behavior include specific upregulation of TrkA receptor in one of the two main ascending nociceptive pathway, i.e., the spinoreticular pathway. Interruption of the NGF signal transduction pathway in laminae V-VI spinal cells may reduce the exacerbated activity of these neurons, then offering an appropriate tool in attempts to alleviate chronic pain. Peripheral NGF sequestration using TrkA-IgG fusion molecules has been showed to reduce acute hyperalgesia induced by carrageenin injection (McMahon et al., 1995). Our study highlights the interest of using TrkA antagonists in the CNS as a therapeutic strategy to alleviate chronic pain.

\section{REFERENCES}

Abbadie C, Besson JM (1992) C-fos expression in rat lumbar spinal cord during the development of adjuvant-induced arthritis. Neuroscience 48:985-993.

Aloe L, Tuveri MA, Carcassi U, Levi-Montalcini R (1992a) Nerve growth factor in the synovial fluid of patients with chronic arthritis. Arthritis Rheum 35:351-355.

Aloe L, Tuveri, MA, Levi-Montalcini R (1992b) Studies on carrageenan-induced arthritis in adult rats: presence of nerve growth factor and role of sympathetic innervation. Rheumatol Int 12:213-216.

Averill S, McMahon SB, Clary DO, Reichardt LF, Priestley JV (1995) Immunocytochemical localization of trkA receptors in chemically identified subgroups of adult rat sensory neurons. Eur J Neurosci 7:1484-1494

Besson JM, Chaouch A (1987) Peripheral and spinal mechanisms of nociception. Physiol Rev 67:67-150.

Butler SH, Weil-Fugazza J, Godefroy F, Besson JM (1985) Reduction of arthritis and pain behaviour following chronic administration of amitriptyline or imipramine in rats with adjuvant-induced arthritis. Pain 23:159-175.

Calvino B, Crepon-Bernard M-O, Le Bars D (1987a) Parallel clinical and behavioural studies of adjuvant-induced arthritis in the rat: possible relationship with "chronic pain." Behav Brain Res 24:11-29.

Calvino B, Villanueva L, Le Bars D (1987b) Dorsal horn (convergent) neurones in the intact anaesthetized arthritic rat. I. Segmental excitatory influences. Pain 28:81-98.

Calvino B, Maillet S, Pradelles P, Besson JM, Couraud JY (1991) Levels of substance P-like immunoreactivity in plasma and cerebral fluid during the course of Freund adjuvant-induced arthritis, a chronic pain model. C R Acad Sci Paris 312:427-432.

Calza L, Pozza M, Zanni M, Manzini CU, Manzini E, Hökfelt T (1997) Peptide plasticity in primary sensory neurons and spinal cord during adjuvant-induced arthritis in the rat: an immunocytochemical and in situ hybridization study. Neuroscience 82:575-589.

Clary DO, Weskamp G, Austin LA, Reichardt LF (1994) TrkA crosslinking mimics neuronal responses to nerve growth factor. Mol Biol Cell 5:549-563.

Coderre TJ, Katz J, Vaccarino AL, Melzack R (1993) Contribution of central neuroplasticity to pathological pain: review of clinical and experimental evidence. Pain 52:259-285.

Colpaert FC (1987) Evidence that adjuvant arthritis in the rat is associated with chronic pain. Pain 28:201-222.

Committee for Research and Ethical Issues of the IASP (1980) Ethical standards for investigations of experimental pain in animals. Pain 9:141-143.

Cook AJ, Woolf CJ, Wall PD, McMahon SB (1987) Dynamic receptive field plasticity in rat spinal horn following $\mathrm{C}$ primary afferent input. Nature 325:151-153.

De Castro Costa M, De Sutter P, Gybels J, Van Hees J (1981) Adjuvantinduced arthritis in rats: a possible animal model of chronic pain. Pain 10:173-185.

Donnerer J, Schuligoi R, Stein C (1992) Increased content and transport of substance $\mathrm{P}$ and calcitonin gene-related peptide in sensory nerves innervating inflamed tissue: evidence for a regulatory function of nerve growth factor in vivo. J Neurosci 49:693-698.

Dougherty PM, Willis WD (1992) Enhanced responses of spinothalamic tract neurons to excitatory amino acids accompany capsaicin-induced sensitization in the monkey. J Neurosci 12:883-894.

Ennulat DJ, Stach RW (1987) Induction of the high-affinity nerve growth factor receptor on embryonic chicken sensory nerve cells by elevated potassium. Neurochem Res 12:839-850.

Ernfors P, Bengzon J, Kokaia Z, Persson H, Lindvall O (1991) Increased levels of messenger RNAs for neurotrophic factors in the brain during kindling epileptogenesis. Neuron 7:165-176.

Gall CM (1993) Seizure-induced changes in neurotophin expression: implication for epilepsy. Exp Neurol 124:150-166.

Gibbs RB, Pfaff DW (1994) In situ hybridization detection of TrkA mRNA in brain: distribution, colocalisation with p75NGFR and upregulation by nerve growth factor. J Comp Neurol 341:324-339.

Guilbaud G, Iggo A, Tegner R (1985) Sensory receptors in ankle joint capsules of normal and arthritic rats. Exp Brain Res 58:29-40.

Guilbaud G, Peschanski M, Briand A, Gautron M (1986) The organization of spinal pathways to ventrobasal thalamus in an experimental model of pain (the arthritic rat). An electrophysiological study. Pain 26:301-312.

Haley JE, Sullivan AF, Dickenson AH (1990) Evidence for spinal $\mathrm{N}$-methyl-D-aspartate receptor involvement in prolonged chemical nociception in the rat. Brain Res 518:218-226.

Holtzman DM, Li YW, Parada LF, Kinsman S, Chen CK, Valletta JS, Zhou J, Long JB, Mobley WC (1992) p140trk messenger RNA marks NGF-responsive forebrain neurons: evidence that trk gene expression is induced by NGF. Neuron 9:465-478.

Jessell TM, Kelly DD (1991) Pain and analgesia. In: Principles of neural science (Kandel ER, Schwartz JH, Jessell TM, eds), pp 385-399. London: Prentice Hall.

Kar S, Gibson SJ, Rees RG, Jura WGZO, Brewerton DA, Polak JM (1991) Increase calcitonin gene-related peptide (CGRP), substance P, and enkephalin immunoreactivities in dorsal spinal cord and loss of CGRP-immunoreactive motoneurons in arthritic rats depend on intact peripheral nerve supply. J Mol Neurosci 3:7-18.

Konttinen YT, Kemppinen P, Serberberg M, Hukkanen M, Rees R, Santavairta S, Sorsa T, Polak JM (1994) Peripheral and spinal neural mechanisms in arthritis, with particular reference to treatment of inflammation and pain. Arthritis Rheum 37:965-982.

Kuraishi Y, Nanayama T, Ohno H, Fujii N, Otaka A, Yajima H, Satoh M (1989) Calcitonin gene-related peptide increases in the dorsal root ganglia of adjuvant arthritic rat. Peptides 10:447-452.

Kuzuna S, Kawai K (1975) Evaluation of analgesic agents in rats with adjuvant arthritis. Chem Pharm Bull 23:1184-1191. 
Lewin GR, Mendell LM (1993) Nerve growth factor and nociception. Trends Neurosci 16:353-359.

Lewin GR, Ritter AM, Mendell LM (1993) Nerve growth factorinduced hyperalgesia in the neonatal and adult rat. $\mathrm{J}$ Neurosci 13:2136-2148.

Lewin GR, Rueff A, Mendell LM (1994) Peripheral and central mechanisms of NGF-induced hyperalgesia. Eur J Neurosci 6:1903-1912.

Lindsay RM, Shooter EM, Radeke MJ, Misko TP, Dechant G, Thoenen H, Lindholm D (1990) Nerve growth factor regulates expression of the nerve growth factor receptor gene in adult sensory neurons. Eur J Neurosci 2:389-396.

McCarthy PW, Lawson SN (1989) Cell type and conduction velocity of rat primary sensory neurons with substance P-like immunoreactivity. Neuroscience 28:745-753.

McCarthy PW, Lawson SN (1990) Cell type and conduction velocity of rat primary sensory neurons with calcitonin gene-related peptide-like immunoreactivity. Neuroscience 34:623-632.

McMahon SB, Bennett DLH, Priestley JV, Shelton DL (1995) The biological effects of endogenous nerve growth factor on adult sensory neurons revealed by a trkA-IgG fusion molecule. Nat Med 1:774-780.

Mendell LM (1996) Neurotrophins and sensory neurons: role in development, maintenance and injury. A thematic summery. Phil Trans R Soc Lond B Biol Sci 351:463-467.

Menétrey D, Besson JM (1982) Electrophysiological characteristics of dorsal horn cells in rats with cutaneous inflammation resulting from chronic arthritis. Pain 13:343-364.

Menétrey D, De Pommery J, Besson JM (1984a) Electrophysiological characteristics of lumbar spinal cord neurons backfired from lateral reticular nucleus in the rat. J Neurophysiol 52:595-611.

Menétrey D, De Pommery J, Roudier F (1984b) Properties of deep spinothalamic tract cells in the rat, with special reference to ventromedial zone of lumbar dorsal horn. J Neurophysiol 52:612-624.

Michael GJ, Kaya E, Averill S, Rattray M, Clary DO, Priestley JV (1997) TrkA immunoreactive neurones in the rat spinal cord. J Comp Neurol 385:441-455.

Molander C, Grant G (1986) Laminar distribution and somatotopic organization of primary afferent fibers from hindlimb nerves in the dorsal horn. A study by transganglionic transport of horseradish peroxidase in the rat. Neuroscience 19:297-312.

Molliver DC, Radeke MJ, Feinstein SC, Snider WD (1995) Presence or absence of TrkA protein distinguishes subsets of small sensory neurons with unique cytochemical characteristics and dorsal horn projections. J Comp Neurol 361:404-416.

Nakane PK (1968) Simultaneous localization of multiple tissue antigens using the peroxidase-labeled antibody method: a study on pituitary glands of the rat. J Histochem Cytochem 16:557-560.
Ness TJ, Gebhart GF (1990) Visceral pain: a review of experimental studies. Pain 41:167-234.

Oku R, Satoh M, Takagi H (1987) Release of substance P from the spinal dorsal horn is enhanced in polyarthritic rats. Neurosci Lett 74:315-319.

Paxinos G, Watson C (1986) The rat brain in stereotaxic coordinates. New York: Academic.

Petty BG, Cornblath DR, Adornato BT, Chaudhry V, Flexner C, Wachsman M, Sinicropi D, Borton LE, Peroutka SJ (1994) The effect of systemically administered recombinant human nerve growth factor in healthy human subjects. Ann Neurol 36:244-246.

Ren K, Hylden JLK, Williams GM, Ruda MA, Dubner R (1992) The effect of a non-competitive NMDA receptor antagonist, MK-801, on behavioural hyperalgesia and dorsal horn neuronal activity in rats with unilateral inflammation. Pain 50:331-344.

Safieh-Garabadian B, Poole S, Allchorne A, Winter J, Woolf CJ (1995) Contribution of interleukin- $\beta$ to the inflammation-induced increase in nerve growth factor levels and inflammation hyperalgesia. Br J Pharmacol 115:1265-1275.

Sobreviela T, Clary DO, Reichardt LF, Brandabur MM, Kordower JH, Mufson EJ (1994) TrkA-immunoreactive profiles in the central nervous system: colocalization with neurons containing $\mathrm{p} 75$ nerve growth factor receptor, choline acetyltransferase, and serotonin. J Comp Neurol 350:587-611.

Swett JE, Woolf CJ (1985) The somatotopic organization of primary afferent terminals in the superficial laminae of the dorsal horn of the rat spinal cord. J Comp Neurol 231:66-77.

Thoenen H (1995) Neurotrophins and neuronal plasticity. Science 270:593-598.

Tonra JR, Curtis R, Wong V, Cliffer KD, Park JS, Timmes A, Nguyen T, Lindsay RM, Acheson A, DiStefano PS (1998) Axotomy upregulates the anterograde transport and expression of brain-derived neurotrophic factor by sensory neurons. J Neurosci 18:4374-4383.

Von Bartheld CS, Byers MR, Williams R, Bothwell M (1996) Anterograde transport of neurotrophins and axodendritic transfers in the developing visual system. Nature 379:830-833.

Winter CA, Kling PJ, Tocco DJ, Tanabe K (1979) Analgesic activity of diflunisal (mk-647;5-(2,4-difluorophenyl)salicyclic acid) in rats with hyperalgesia. J Pharmacol Exp Ther 211:625-678.

Woolf CJ, McMahon SB (1985) Injury-induced plasticity of the flexor reflex in chronic decerebrate rats. Neuroscience 16:395-404.

Wyatt S, Davies M (1993) Regulation of expression of mRNAs encoding the nerve growth factor receptors p75 and trkA in developing sensory neurons. Development 119:635-647. 